\title{
A Case of Metastatic Extramammary Paget's Disease Responding to Trastuzumab plus Paclitaxel Combination Therapy
}

\author{
Fumie Hanawa Takashi Inozume Kazutoshi Harada \\ Tatsuyosi Kawamura Naotaka Shibagaki Shinji Shimada \\ Department of Dermatology, University of Yamanashi, Chuo, Japan
}

\section{Key Words}

Extramammary Paget's disease - Human epidermal growth factor receptor 2 .

Trastuzumab · Paclitaxel

\begin{abstract}
There is no effective treatment for advanced extramammary Paget's disease (EMPD). The human epidermal growth factor receptor 2 (HER2) protein is often overexpressed in EMPD. Trastuzumab is a humanized monoclonal antibody against HER2 used in the treatment of breast cancers in which HER2 is overexpressed. We report a case of advanced EMPD in which trastuzumab and paclitaxel combination therapy was effective. The patient was a 70-year-old Japanese woman who presented with EMPD on the vulva and multiple metastatic lymph nodes. Immunohistochemical staining revealed strong HER2 protein expression in the primary tumor and metastatic lymph nodes. The patient received trastuzumab and paclitaxel. After 4 courses of this regimen, the mass on the vulva and the metastatic lymph nodes regressed. Our findings may imply that trastuzumab plus paclitaxel combination therapy is useful for the treatment of advanced EMPD overexpressing HER2.
\end{abstract}

\section{Introduction}

Extramammary Paget's disease (EMPD) is a relatively rare malignant skin tumor which is considered to originate from cutaneous appendages and occurs in cutaneous areas bearing apocrine glands, such as the scrotum, vulva, and axilla. Generally, its prognosis is favorable if the primary lesion is completely resected and no metastases are present. However, the prognosis of the progressive disease is poor because there is currently no effective treatment for advanced EMPD. 
Trastuzumab (Herceptin ${ }^{\circledR}$; Genentech, Inc., San Francisco, Calif., USA) is a humanized monoclonal antibody which selectively targets human epidermal growth factor receptor 2 (HER2) and is approved for the treatment of metastatic breast cancer (MBC). Trastuzumab used in combination with paclitaxel is the first-line chemotherapy for HER2-overexpressing MBC, with a response rate of 56-84\% [1, 2]. Similarly, the HER2 protein is sometimes overexpressed in EMPD, and in such cases trastuzumab plus paclitaxel combination therapy is also expected to be effective.

We describe a case of advanced EMPD in which the primary tumor mass and lymph node metastasis regressed with combined trastuzumab and paclitaxel therapy. Our observation may imply that this combination therapy is effective for advanced EMPD with HER2 overexpression.

\section{Case Report}

A 70-year-old Japanese woman presented with a 10-year history of an asymptomatic erythema on the vulva, which had gradually increased in size. She was referred to our hospital in October 2008. Physical examination revealed a $10 \times 13 \mathrm{~cm}$, slightly raised erythematous lesion with ulcers and a

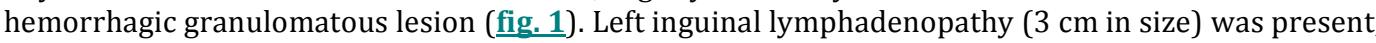
but without tenderness. Computed tomography (CT) and positron emission tomography revealed multiple lymph node swelling in the pararenal artery lymph node, the left inguinal lymph node and the subclavian axillary lymph node. Histological examination of biopsy specimens taken from the genital area and left inguinal lymph node showed atypical tumor cells with an abundant pale-staining cytoplasm and large atypical nuclei (fig. 2a). On the basis of these findings, we made a diagnosis of advanced EMPD. Immunohistochemical staining showed that the Paget's cells stained strongly for the Her2 protein (fig. 2b). The role of the HER2 signal pathway in EMPD was assessed. Epidermal growth factors bind to HER2 receptors, and this signal is transduced into the phosphatidylinositol 3 kinase and extracellular signal-regulated kinase (ERK) pathways by phosphorylation of AKT (pAKT) and ERK (pERK). Activation of pAKT and pERK is considered to be a hallmark of the activation of these signal transduction pathways [3]. Immunohistochemical staining of the biopsy specimens of the primary tumor revealed that Paget's cells were positive for both pAKT and pERK (fig. 2c, d).

These results suggested that trastuzumab might be effective for the present HER2-overexpressing EMPD case. Therefore, we initially decided to treat this patient with trastuzumab. After gaining the informed consent of the patient, we administered trastuzumab at a loading dose of $4 \mathrm{mg} / \mathrm{kg}$ intravenously. After the first dose, a weekly $2-\mathrm{mg} / \mathrm{kg}$ maintenance dose was administered for 5 weeks. However, there was no objective response. We then treated the patient with weekly trastuzumab $(2 \mathrm{mg} / \mathrm{kg})$ in combination with paclitaxel $(80 \mathrm{mg} / \mathrm{kg})$. This combination therapy was administered weekly for 6 weeks followed by a 2-week rest according to a protocol for HER2-positive MBC. After 4 courses of this therapy, the granulomatous lesion on the vulva had disappeared (fig. 1b). A CT scan also revealed the regression of the pararenal artery lymph node metastasis ( $\underline{\text { fig. } 3}$ ). Histopathologically, most of the tumor cells in the epidermal nest and lymph nodes had disappeared. Similarly, almost no Paget's cells overexpressing the HER2 protein could be identified (fig. $2 \mathrm{f}$ ). After 6 courses, we stopped the combination therapy because the patient developed grade 3 neutrophilic leukopenia. In December 2009, a remarkable edema of the left leg appeared, and enlargement of the left inguinal lymph nodes was detected by CT. Since then, the patient has been treated with local irradiation for both the inguinal lymph nodes and the primary lesion. 


\section{Discussion}

The prognosis of advanced EMPD is extremely poor, and there is currently no effective treatment for this condition. Several studies have shown frequent amplification of the HER2 gene and overexpression of the HER2 protein in patients with EMPD. It has been reported that $20-60 \%$ of EMPD patients express the HER2 protein [4-7]. Trastuzumab, a humanized monoclonal antibody against HER2, has found to be useful in treating MBC. Targeted treatment of HER2-positive MBC with single-agent trastuzumab demonstrates favorable efficacy, and this efficacy is enhanced by the combination with paclitaxel $[1,2,8,9]$. Therefore, combination treatment with trastuzumab and paclitaxel is used as a first-line standard therapy for HER2-positive MBC.

In our case, the patient had multiple lymph node metastases, and we had considered her as inoperable. Both the primary site and the metastatic lymph nodes expressed the HER2 protein, pAKT and pERK, as determined by immunohistochemical staining (fig. $2 b-d)$. Activation of pAKT and pERK is a hallmark of the activation of HER2 signal transduction pathways. Ogawa et al. [3], using immunohistochemical staining, reported that some cases of EMPD presented with overexpression of the HER2 protein, pAKT and pERK. They showed that pAKT was detected in $75.0 \%$ and pERK in $38.9 \%$ of EMPD patients. Both the phosphatidylinositol 3 kinase and the ERK pathways appear to be involved in the progression of EMPD. It has been postulated that antibody-dependent cellular cytotoxicity may play an important role in the mechanism of action of trastuzumab [10]. On the other hand, trastuzumab binds to the surfaces of HER2overexpressing cancer cells and inhibits downstream signaling via pAKT and pERK. In our case, after combination therapy with trastuzumab and paclitaxel, the expressions of the HER2 protein, pAKT and pERK in EMPD were decreased (fig. $2 \mathrm{f}-\mathrm{h}$ ). These results may imply that paclitaxel plus trastuzumab combination therapy selectively targets the tumor cells with high HER2 expression or that it decreased the signal transduction of HER2.

In this particular case, the combination therapy with trastuzumab and paclitaxel, the standard first-line chemotherapy for MBC, was effective for progressive-stage EMPD with HER2 overexpression. Our observations may suggest that this therapy should be considered as an option for the treatment of HER2-positive EMPD.

\section{Disclosure Statement}

The authors have no conflict of interest to declare. 

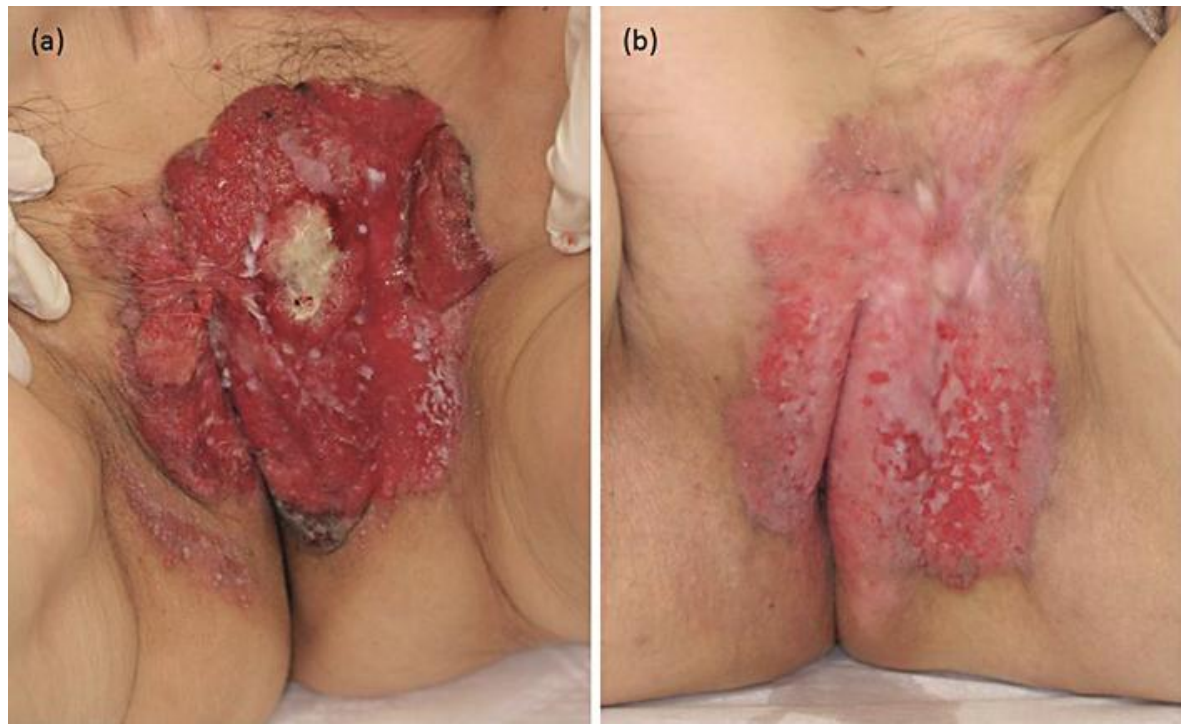

Fig. 1. a A granulomatous lesion with ulcers on the vulva. b A scar on the vulva after 6 courses of paclitaxel plus trastuzumab combination therapy.

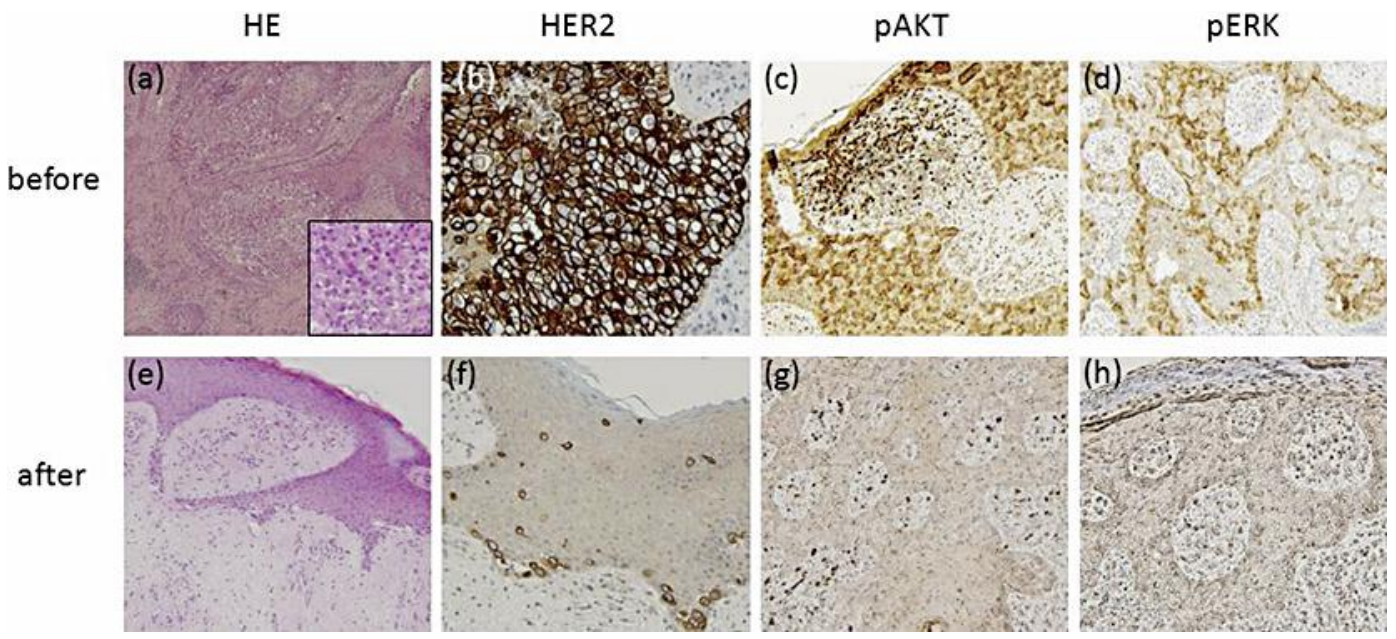

Fig. 2. Microscopic examination revealed numerous nests of Paget's cells in the epidermis before the therapy (a); however, the number of these cells decreased after the therapy (e). HE stain; original magnification, $\times 100$. Representative results for the immunohistochemical examination of HER2, pAKT and pERK before $(\mathbf{b}-\mathbf{d})$ and after $(\mathbf{f}-\mathbf{h})$ the therapy. 

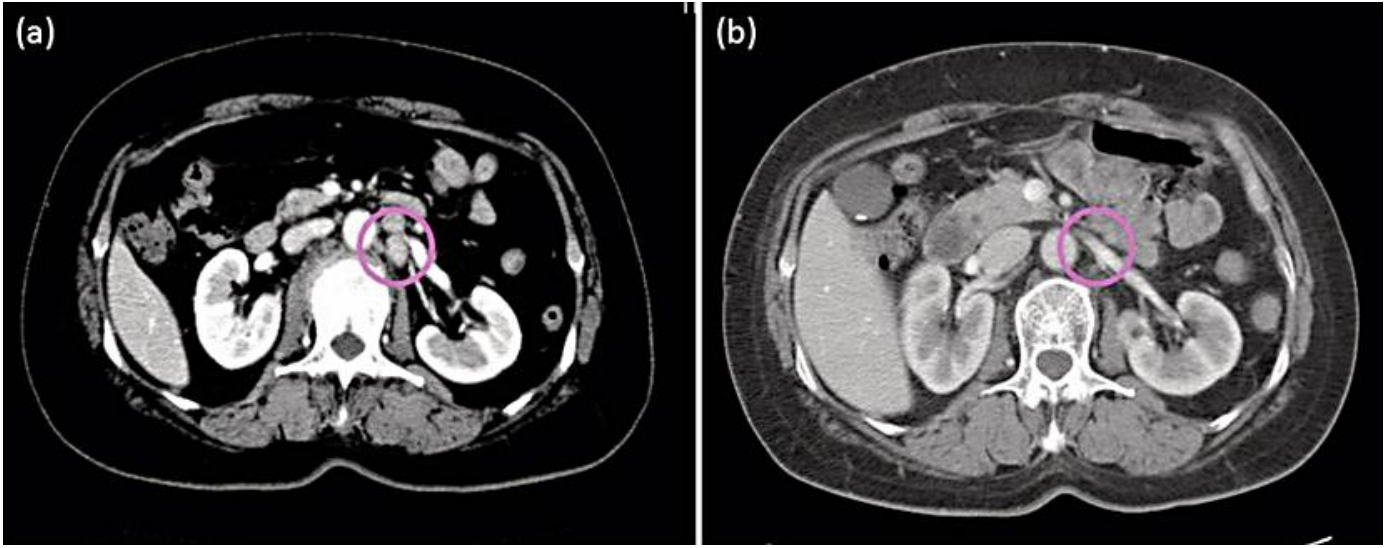

Fig. 3. a A swollen pararenal artery lymph node identified using CT. b Apparent regression of the metastatic lesion in the lymph node after trastuzumab plus paclitaxel administration. Circles indicate lymph nodes.

\section{References}

1 Perez EA, Suman VJ, Rowland KM, Ingle JN, Salim M, Loprinzi CL, Flynn PJ, Mailliard JA, Kardinal CG, Krook JE, Thrower AR, Visscher DW, Jenkins RB: Two concurrent phase II trials of paclitaxel/carboplatin/trastuzumab (weekly or every-3-week schedule) as first-line therapy in women with HER2-overexpressing metastatic breast cancer: NCCTG study 983252. Clin Breast Cancer 2005;6:425-432.

-2 Burris H 3rd, Yardley D, Jones S, Houston G, Broome C, Thompson D, Greco FA, White M, Hainsworth J: Phase II trial of trastuzumab followed by weekly paclitaxel/carboplatin as first-line treatment for patients with metastatic breast cancer. J Clin Oncol 2004;22:1621-1629.

-3 Ogawa T, Nagashima Y, Wada H, Akimoto K, Chiba Y, Nagatani T, Inayama Y, Yao M, Aoki I, Ikezawa Z: Extramammary Paget's disease: analysis of growth signal pathway from the human epidermal growth factor receptor 2 protein. Hum Pathol 2005;36:1273-1280.

4 Meissner K, Rivière A, Haupt G, Löning T: Study of neu-protein expression in mammary Paget's disease with and without underlying breast carcinoma and in extramammary Paget's disease. Am J Pathol 1990;137:1305-1309.

5 Wolber RA, Dupuis BA, Wick MR: Expression of c-erbB-2 oncoprotein in mammary and extramammary Paget's disease. Am J Clin Pathol 1991;96:243-247.

-6 Keatings L, Sinclair J, Wright C, Corbett IP, Watchorn C, Hennessy C, Angus B, Lennard T, Horne CH: c-erbB-2 oncoprotein expression in mammary and extramammary Paget's disease: an immunohistochemical study. Histopathology 1990;17:243-247.

7 Tanskanen M, Jahkola T, Asko-Seljavaara S, Jalknen J, Isola J: HER2 oncogene amplification in extramammary Paget's disease. Histopathology 2003;42:575-579.

-8 Cobleigh MA, Vogel CL, Tripathy D, Robert NJ, Scholl S, Fehrenbacher L, Wolter JM, Paton V, Shak S, Lieberman G, Slamon DJ: Multinational study of the efficacy and safety of humanized anti-HER2 monoclonal antibody in women who have HER2-overexpressing metastatic breast cancer that has progressed after chemotherapy for metastatic disease. J Clin Oncol 1999;17:2639-2648.

99 Vogel CL, Cobleigh MA, Tripathy D, Gutheil JC, Harris LN, Fehrenbacher L, Slamon DJ, Murphy M, Novotny WF, Burchmore M, Shak S, Stewart SJ, Press M: Efficacy and safety of trastuzumab as a single agent in first-line treatment of HER2-overexpressing metastatic breast cancer. J Clin Oncol 2002;20:719-726.

10 Clynes RA, Towers TL, Presta LG, Ravetch JV: Inhibitory Fc receptors modulate in vivo cytoxicity against tumor targets. Nat Med 2000;6:443-446. 